\title{
Taguchi Yöntemleri Kullanılarak Frezeleme İşleminde Kesme Parametrelerinin Yüzey Pürüzlülügüüne Etkisinin Araştırılması
}

\author{
Ali Mamedov ${ }^{1}$
}

\section{ÖZ}

Bu çalışmada, kalıp çeliğinin işlenmesi esnasında kesme parametrelerinin yüzey pürüzlülüğü üzerindeki etkisinin araştırılması yoluyla frezeleme operasyonunun optimizasyonu amaçlanmıştır. Deneysel çalışmaların sonuçları, kritik parametrelerin bulunmasında etkili olan sinyal-gürültü oranı hesaplaması ve varyans analizi gibi farklı Taguchi yöntemleri kullanılarak değerlendirilmiştir. Elde edilen S/G oranlarına bakılarak yüzey pürüzlülüğünün sırasıyla radyal dalma oranı, kesme derinliği, iş mili devri ve ilerleme hızından etkilendiğini söylemek mümkün. Bu kesme parametreleri için optimum değerler $16 \mathrm{~mm} /$ dak ilerleme hızı, $200 \mathrm{dev} /$ dak iş mili devri, $0.35 \mathrm{~mm}$ kesme derinliği ve $\% 50$ radyal dalma oranı olarak hesaplanmıştır. Bu kesme parametrelerinde yapılan doğrulama deneyi işlenmiş yüzeyin pürüzlülüğünün $\mathrm{Ra}=0.09 \mu \mathrm{m}$ kadar düştügünü göstermiştir.

Anahtar Kelimeler: Frezeleme işlemi, yüzey pürüzlülüğü, taguchi yöntemleri

\section{Investigation of the Effect of Cutting Parameters on Surface Roughness During Milling Operation Using Taguchi Methods}

\begin{abstract}
This work is devoted to the optimization of milling operation by determination of the effect of cutting parameters on surface roughness throughout the machining of the mold steel. The experimental studies results are presented by applying different Taguchi methods, such as signal to noise ratio calculation and analysis of variance, which are considered effective in finding the critical parameters and their contributions. From obtained results of the $\mathrm{S} / \mathrm{N}$ responses it can be concluded that the surface roughness was influenced by radial tool immersion, depth of cut, spindle speed and feed rate, respectively. The optimal values for cutting parameters were calculated as $16 \mathrm{~mm} / \mathrm{min}$ for feedrate, $200 \mathrm{rpm}$ for spindle speed, $0.35 \mathrm{~mm}$ for depth of cut and $\% 50$ for radial tool immersion. Experimental results of the milling operation with these parameters showed that average surface roughness reduced up to $\mathrm{Ra}=0.09 \mu \mathrm{m}$.
\end{abstract}

Keywords: Milling process, surface roughness, taguchi methods

\footnotetext{
* Iletişim Yazarı

Geliş/Received

18.11.2020

Kabul/Accepted

: 22.03.2021

1 College of Engineering and Technology, American University of the Middle East, Kuwait

ali.mamedov@aum.edu.kw, ORCID: 0000-0003-0560-552X
} 


\section{EXTENDED ABSTRACT}

\section{Introduction}

This work is devoted to the optimization of milling operation by determination of the effect of cutting parameters on surface roughness throughout the machining of the mold steel. Significant amount of research was dedicated to find optimal milling conditions resulting in low surface roughness at high material removal rates. Some of the researcher also investigated the effect of the chatter vibrations on surface roughness and suspension methods to eliminate them. In this paper the experimental studies results are presented by applying different Taguchi methods, such as signal to noise ratio calculation and analysis of variance, which are considered effective in finding the critical parameters and their contributions. The effects of four cutting parameters on surface roughness are evaluated, including feedrate, spindle speed, depth of cut and radial tool immersion.

\section{Objectives}

The main objective of this paper is to investigate and evaluate the roughness of generated surface after milling of hardened mold steel to find the optimum level of the selected parameters and their contribution via Taguchi methods. Four controllable cutting parameters, namely the feed rate, the spindle speed, the depth of cut, and radial tool immersion, are chosen to evaluate their effect on surface roughness.

\section{Methods}

Signal to Noise ratio method was used to reduce the noise of experimental data based on different definitions of quality characteristics. Method contains three types of quality characteristics, namely, the smaller the better, the nominal the best, and the larger the better. Considering the main objective of this study, the smaller the better characteristic of $\mathrm{S} / \mathrm{N}$ ratio will be used in order to reduce surface roughness of machined surface. Once, significant parameters affecting the surface roughness and their optimum levels are determined, analysis of variance methodology will be used to determine the percentage of their contributions. Based on the number of selected parameters and their levels, L9 orthogonal array of Taguchi was selected. This array will be used to evaluate effect of all parameters with minimum number of experiments. Consequently, nine experiments were implemented in order to evaluate the effect of the controllable cutting paramenters on surface roughness of mold steel. The contact type profilometer was used to measure the average surface roughness (Ra). Measurements were repeated three time on different regions of the surface to ensure precision and accuracy of the results. The response values were evaluated according to the average $\mathrm{S} / \mathrm{N}$ ratio to find optimal levels of selected parameters. Finally, cutting experiment with determined optimal cutting parameters was performed and surface roughness improvement was evaluated.

\section{Results and Conclusions}

From obtained results of the $\mathrm{S} / \mathrm{N}$ responses it can be concluded that the highest $\mathrm{S} / \mathrm{N}$ ratios of individual parameters of different levels represent the most optimal level of each parameter. Additionally, the highest difference between the maximum value and minimum value of $\mathrm{S} / \mathrm{N}$ ratio for each parameter represents the most significant factor affecting surface roughness. It has been seen that the surface roughness was influenced by radial tool immersion, depth of cut, spindle speed and feed rate, respectively. The optimal values for cutting parameters were calculated as $16 \mathrm{~mm} / \mathrm{min}$ for feedrate, $200 \mathrm{rpm}$ for spindle speed, $0.35 \mathrm{~mm}$ for depth of cut and $\% 50$ for radial tool immersion. Experimental results of the milling operation with these parameters showed that average surface roughness reduced up to $\mathrm{Ra}=0.09 \mu \mathrm{m}$. The results of the analysis of variance determined the percentage of contribution for each individual parameters as $49.12 \%$ for radial tool immersion, $23.67 \%$ for spindle speed, $20.23 \%$ for depth of cut and $6.98 \%$ for feedrate. Therefore, it can be concluded that radial tool immersion has the highest influence on generated surface for these experiments. The implementation of the optimal parameters experimentally validated that generated surface had the smallest surface roughness among all configurations of cutting parameters. 


\section{GIIRIŞ}

Günümüzün rekabetçi endüstrisinde, süper alaşımlar ve sertleştirilmiş çelikler gibi yüksek performanslı malzemelere talep vardır. Frezeleme işlemleri, havacılık, biyomedikal ve otomotiv gibi farklı endüstriyel sektörlerde bahsedilen yüksek performanslı malzemeler kullanılarak karmaşık geometrili parçaların üretiminde yaygın olarak kullanılmaktadır. Frezeleme işlemi sırasında yüzey oluşum mekanizmasının anlaşılması ve nihai yüzey pürüzlülüğünün öngörülmesi, deneme yanılma yöntemlerine başvurmadan istenen yüzey kalitesini elde etmek için son derece önemlidir. Aynı zamanda istenilen yüzey kalitesine en hızlı şekilde ulaşmak, üretim maliyetini düşürecek ve üretim verimliliğini artıracaktır. Bu nedenle, yüzey topografisini modellemek ve frezeleme işlemleri sırasında oluşacak yüzey pürüzlülüğünü öngörmek için önemli miktarda araştırma yapılmıştır. Montgomery ve Altıntaş [1] kaldırılacak talaş kalınlığını hesaplamak için kesici ucun trokoidal hareketini dikkate alan model geliştirmişlerdir. Bu model kesici ucun ve iş parçasının kinematiğini dikkate alarak işleme sonrasında oluşacak yüzeyi öngörmektedir. Ehmann ve Hong [2] yüzey modellemesini daha da ileriye taşıyarak kesme işlemi esnasında oluşabilecek takım sapması, işleme tezgâhı deformasyonu ve titreşimi gibi hataların da yüzey pürüzlülüğü üzerinde etkilerini araştırmışlardır.

Günümüzde karmaşık geometrili parçaların üretilebilmesi için işleme süreçlerinin daha karmaşık hale gelmesi ile beraber yüzey pürüzlülüğünü öngüren modellerin de bunlara ayak uydurması gerekmektedir. Layegh ve Lazoğlu [3] 5-eksenli frezeleme işlemi için üç boyutlu yüzey topografisini öngören model geliştirmişlerdir. Bahse konu model çeşitli takım açılarında işleme esnasında oluşan yüzey topografisini ve pürüzlülüğünü öngörmektedir. Frezeleme işlemi esnasında tırlama titreşimlerinin etkileri Altıntaş ve Budak [4] tarafından analitik olarak modellenmiştir. Munoa vd. [5], metal kesme işlemlerinde tırlama titreşimleri için süspansiyon teknikleri üzerine kapsamlı bir eleştirel derleme yayınlamıştır. Zahoor vd. [6] dikey frezeleme sırasında zorlanmış titreşimlerin AISI P20 malzemesinin yüzey pürüzlülüğü üzerindeki etkisini analiz etmiş ve titreşim genliği ve kesme derinliğinin yüzey pürüzlülüğünü etkileyen en önemli iki faktör olduğunu belirtmiştir. Ayrıca yazarlar, aşırı tezgah titreşimlerinin kesici takım kırılmalarına da neden olduğunu belirtmişlerdir. Bağc1 ve Aykut [7], ilerleme hızı, kesme hızı ve kesme derinliğinin kobalt bazlı alaşımın optimum yüzey pürüzlülüğü üzerindeki etkisini Taguchi optimizasyon yöntemini kullanarak bir dizi frezeleme deneyleri aracılığıyla analiz etmişlerdir. Kuram ve Özçelik [8] takım aşınmasını, kesme kuvvetlerini ve yüzey pürüzlülüğünü optimize etmek için Taguchi’nin sinyal-gürültü oranı yöntemini kullanmışlar. Costa vd. [9], AISI 1045 çeliğinin soğutma sıvısı kullanmadan frezelemesi sırasında maksimum malzeme kaldırma oranı ve minimum yüzey pürüzlülüğü ile sonuçlanacak optimum kesme parametrelerini belirlemek için Taguchi'nin sinyal-gürültü oranı yöntemiy- 
le birlikte Ağırlıklı Temel Bileşen Analizi yöntemini kullanmışlar. Manav vd. [10], takım yolu optimizasyon algoritmasına dayalı olarak işlenmiş yüzeyler için yüzey pürüzlülüğü optimizasyonu modeli önermiştir. Bahsedilen model, eşzamanlı olarak kesme kuvveti, sivri uç (scallop) yüksekliği ve işleme süresini en aza indirme gibi birden çok hedefi dikkate almaktadır. Süper alaşımlar ve sertleştirilmiş çelikler gibi yüksek performanslı malzemelerin işlenmesi sırasında ek komplikasyonlar ortaya çıkar. Arruda vd. [11], bitirme işlemi sırasında AISI H13 sıcak iş takım çeliğinin yüzey pürüzlülüğünü öngörmek için optimizasyon modelinde kontrol parametreleri olarak diş başına ilerleme, eksenel kesme derinliği, radyal kesme derinliği ve kesme hızı kullanmıştır. Mia vd. de [12] sertleştirilmiş AISI 4140 çeliğinin frezelenmesi sırasında minimum kesme kuvveti ve yüzey pürüzlülüğü ile sonuçlanacak MQL (Minimum Miktar Yağlama) için optimum sıvı akış oranını bulmak için Taguchi tabanlı Gray ilişkisel analizini kullanmıştır.

Yukarıda sunulan çalışmalarda, yüksek sertliğe ve aşınma dayanımına sahip malzemelerin işlemesi sırasında kesme parametrelerinin yüzey kalitesini önemli ölçüde etkilediğini açıkça belirtilmektedir. Mevcut literatürde yüzey pürüzlülüğü matematiksel model veya sonlu elemanlar yöntemi kullanılarak öngörülmektedir. Onlardan farklı olarak, bu makalenin temel amac1, minimum yüzey pürüzlülüğünü verecek kesme parametrelerin optimum seviyesini Taguchi yöntemlerini kullanarak tespit etmek ve sertleştirilmiş kalıp çeliği Hardox 600 malzemesi üzerinde doğrulamaktır. Sadece deneysel verilere dayanarak, diğer modellere nazaran daha hızlı seçilebilen optimum ilerleme hızı, iş mili devri, kesme derinliği ve radyal dalma oranı parametreleri endüstriyel uygulamalar için daha kullanışlıdır.

\section{YÖNTEM VE ÖLÇÜMLER}

Çalışmanın amacı, işlenmesi güç kalıp çeliğinden imal edilen parçaların yüzey pürüzlülüğünü etkileyen kesme parametrelerinin ve seviyelerinin en iyi kombinasyonunu belirleyerek yüzeyin kalitesini iyileştirmektir. Bu çalışmada, Tablo 1'de gösterildiği gibi, dört kesme parametresi üç farklı değerde incelenmiştir. Bunlar: $\left(P_{1}\right)$ - ilerleme hızı (mm/dak), $\left(P_{2}\right)$ - iş mili devri (devir/dak), $\left(P_{3}\right)$ - kesme derinliği $(\mathrm{mm})$ ve $\left(P_{4}\right)$ radyal dalma oranı $(\%)$ şeklindedir. Bu değerlerin seçimi, çok çeşitli endüstriyel uy-

Tablo 1. Seçilen Parametreler ve Değerleri

\begin{tabular}{|l|c|c|c|c|}
\hline & $\begin{array}{c}\boldsymbol{P}_{1} \text { : Ilerleme hızı } \\
\text { (mm/dak) }\end{array}$ & $\begin{array}{c}\boldsymbol{P}_{2} \text { : Iş mili devri } \\
\text { (devir/dak) }\end{array}$ & $\begin{array}{c}\boldsymbol{P}_{3} \text { : Kesme derinliği } \\
(\mathbf{m m})\end{array}$ & $\begin{array}{c}\boldsymbol{P}_{4} \text { : Radyal dalma oranı } \\
(\%)\end{array}$ \\
\hline Değer 1 & 16 & 200 & 0.2 & 33 \\
\hline Değer 2 & 25 & 250 & 0.35 & 50 \\
\hline Değer 3 & 40 & 315 & 0.5 & 100 \\
\hline
\end{tabular}


gulamalara dayanmaktadır. Taguchi ortogonal dizi yöntemi, frezeleme sürecini optimize etmek ve parametrelerin en iyi konfigürasyonunu elde etmek için kullanılmıştır.

Deneyler maksimum iş mili devri 800 devir/dak ve gücü $7.5 \mathrm{~kW}$ olan konvansyonel üç eksenli freze tezgâhında, $100 \mathrm{~mm}$ çapında freze kafasına dört adet TiCN kap11 karbür kesme ucu takılarak yapılmıştır. Bu çalışma için iş parçası olarak sertliği 570 Brinnel, akma dayanımı $1650 \mathrm{MPa}$ ve çekme dayanımı $2000 \mathrm{MPa}$ olan su verilmiş ve temperlenmiş, aşınmaya dayanıklı Hardox 600 takım çeliği seçilmiştir. Yüzey pürüzlülüğünü $(\mathrm{Ra})$ ölçümleri için Surfcam 200 temas tipi yüzey pürüzlülüğü ölçüm cihazı kullanılmıştır. Şematik deney düzeneği Şekil-1'de gösterilmiştir. Ölçüm prosedürünün doğruluğunun ve hassasiyetinin elde edilmesini sağlamak için, yüzey pürüzlülüğü ölçümü işlenmiş yüzeylerin farklı noktalarında üç kez tekrarlanmıştır. Hesaplamaların bir sonraki adımlarında bu üç ölçüm değerinin ortalaması olan yüzey pürüzlülüğü değeri kullanılmıştır.

Kontrol edilebilir parametrelerin sayısına ve seviyelerine bağlı olarak, Tablo 2'de gösterildiği gibi, Taguchi’nin L9 ortogonal dizisi seçilmiştir. Seçilen ortogonal dizinin

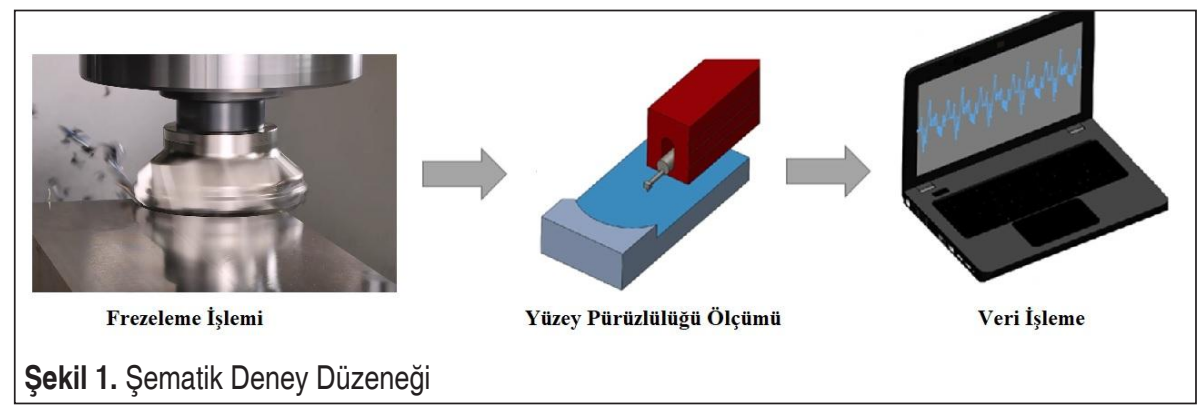

Tablo 2. Veri Toplama Için Taguchi’nin L9 Ortogonal Dizisi

\begin{tabular}{|c|c|c|c|c|}
\hline $\begin{array}{c}\text { Deney } \\
\#\end{array}$ & $\begin{array}{c}\boldsymbol{P}_{1} \text { : ilerleme hızı } \\
(\mathbf{m m} / \text { dak) }\end{array}$ & $\begin{array}{c}\boldsymbol{P}_{2}: \text { iş mili devri } \\
\text { (dev/dak) }\end{array}$ & $\begin{array}{c}\boldsymbol{P}_{3}: \text { Kesme derin- } \\
\text { liği }(\mathbf{m m})\end{array}$ & $\begin{array}{c}\boldsymbol{P}_{4}: \text { Radyal dalma } \\
\text { oranı }(\%)\end{array}$ \\
\hline 1 & 16 & 200 & 0.2 & 33 \\
\hline 2 & 16 & 250 & 0.35 & 50 \\
\hline 3 & 16 & 315 & 0.5 & 100 \\
\hline 4 & 25 & 200 & 0.35 & 100 \\
\hline 5 & 25 & 250 & 0.5 & 33 \\
\hline 6 & 25 & 315 & 0.2 & 50 \\
\hline 7 & 40 & 200 & 0.5 & 50 \\
\hline 8 & 40 & 250 & 0.2 & 100 \\
\hline 9 & 40 & 315 & 0.35 & 33 \\
\hline
\end{tabular}



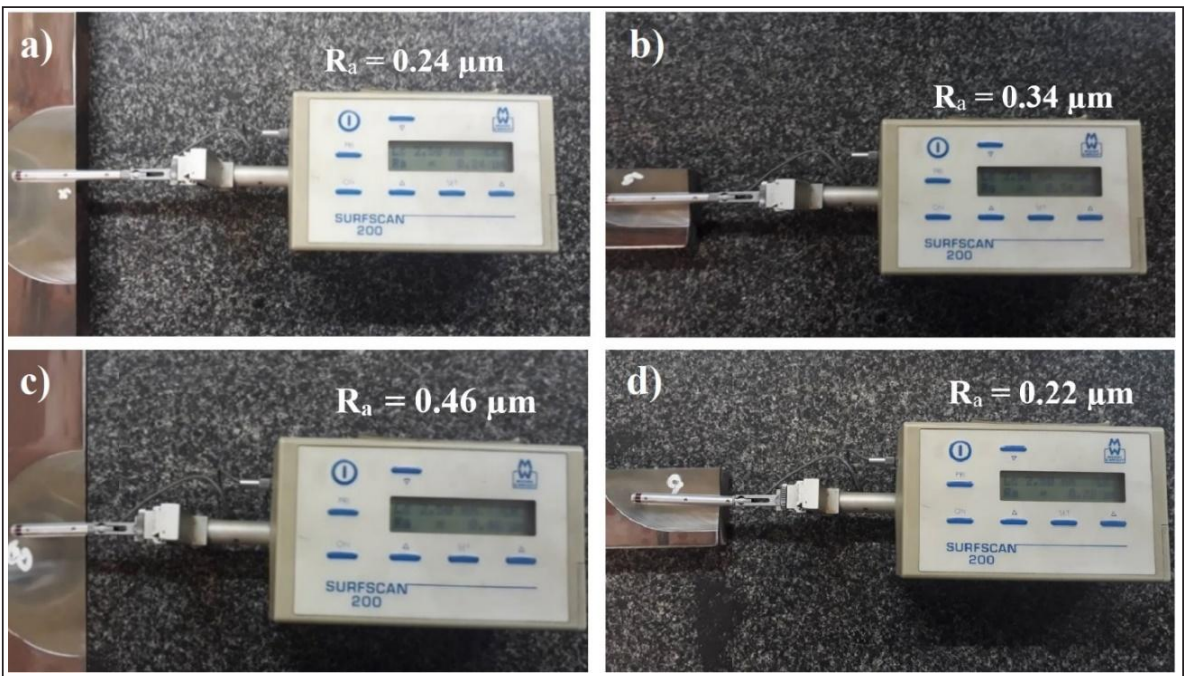

Şekil 2. Numune Yüzey Pürüzlülüğü Ölçümleri: a) Deney 4, b) Deney 5, c) Deney 8, d) Deney 9

bu tasarımı, az sayıda deneyle tüm parametreleri etkisini değerlendirme imkânı sunar.

Her deney numunesi için kesme parametreleri Tablo-2'de gösterildiği değerlerde ayarlanarak işlendiğinden sonra, Şekil-2'de gösterildiği gibi yüzey pürüzlülüğü ölçümleri alınmıştır.

\subsection{Sinyal Gürültü Oranı}

Taguchi, mühendislik tasarımında herhangi bir problem için dikkate alınan kalite özelliklerini belirlemek için Sinyal Gürültü (S/G) oranının hesaplanmasını önerir. S/G oranının üç kategorisi vardır: "en küçük olan en iyi”, "nominal olan en iyi" ve "en yüksek olan en iyi" [13]. S/G oranı, karşılaştırma ve değerlendirme işlemi için deney sonuçlarını tek bir değere dönüştürecektir. Çalışanın amacına uygun olarak yüzey pürüzlülüğünü en aza indirmek için, Denklem-1'de gösterildiği gibi, S/G oranının hesaplanması için “en küçük olan en iyi” kategori seçilmişstir.

$$
S / G=-10 \log \left(\frac{1}{N}\left(\sum_{i=1}^{n} y_{i}^{2}\right)\right)
$$

burada, $y_{1}$ farklı deneylerin yüzey pürüzlülüğünü, $N$ ise her deney için toplam veri noktasını temsil eder. Ölçülen ortalama yüzey pürüzlülüğü değerleri ve Denklem-1 kullanılarak hesaplan S/G oranları Tablo 3’te gösterilmiştir.

Tablo 3 'teki toplanan verilerden, kontrol edilebilir parametrelerin optimal seviyelerini 
Tablo 3. Tüm Deneyler Için S/G Oranı

\begin{tabular}{|c|c|c|}
\hline Deney \# & Ortalama yüzey pürüzlülüğü $R_{a}(\mu \mathrm{m})$ & $S / G$ oranı \\
\hline 1 & 0.14 & 17.07 \\
\hline 2 & 0.14 & 17.07 \\
\hline 3 & 0.40 & 7.95 \\
\hline 4 & 0.24 & 12.39 \\
\hline 5 & 0.34 & 9.37 \\
\hline 6 & 0.24 & 12.39 \\
\hline 7 & 0.20 & 13.97 \\
\hline 8 & 0.46 & 6.74 \\
\hline 9 & 0.22 & 13.15 \\
\hline
\end{tabular}

bulmak için Tablo 4'te gösterildiği gibi ortalama S/G oranlarının yanıtları hesaplanmıştır.

Tablo 4. S/G Oranlarının Yanıt Tablosu

\begin{tabular}{|c|c|c|c|c|}
\hline & $\begin{array}{c}P_{1}: \text { ilerleme } \\
\text { hızı }\end{array}$ & $\begin{array}{c}P_{2}: \text { İş mili } \\
\text { devri }\end{array}$ & $\begin{array}{c}P_{3}: \text { Kesme } \\
\text { derinliği }\end{array}$ & $\begin{array}{c}P_{4}: \text { Radyal dalma } \\
\text { oranı }\end{array}$ \\
\hline Değer 1 & 14.03 & 14.48 & 12.07 & 13.19 \\
\hline Değer 2 & 11.38 & 11.06 & 14.20 & 14.48 \\
\hline Değer 3 & 11.29 & 11.16 & 10.48 & 9.03 \\
\hline$|\Delta \mathrm{T}|$ & 2.74 & 3.41 & 3.77 & 5.45 \\
\hline
\end{tabular}

Tablo 4'te $|\Delta \mathrm{T}|$ maksimum değerle minimum değerin arasındaki farkı gösterir. $|\Delta \mathrm{T}|$ 'nin büyüklük değeri Tablo 4'te gösterilen her bir parametrenin önemini belirtmektedir. Bu deneyler için $P_{4}$ 'ün en önemli parametre olduğu açıktır ve bunu $P_{3}, P_{2}$ ve $P_{1}$ takip eder. Optimum parametre seti, her parametre için en yüksek $\mathrm{S} / \mathrm{G}$ seviyesi seçilerek Tablo 4'ten değerlendirilebilir. En iyi sonuçlar için ilerleme hızı 16 mm/dak, iş mili devri 200 devir/dak, kesme derinliği $0.35 \mathrm{~mm}$ ve radyal dalma oranı \%50 olarak seçilmelidir. $\mathrm{Bu}$ parametrelerle yapılan frezeleme işlemi sonucunda elde edilen işparçasının ortalama yüzey pürüzlülüğü, Şekil 3'te gösterildiği gibi, $\mathrm{R}_{\mathrm{a}}=0.09 \mu \mathrm{m}$ olarak ölçülmüştür.

Şekil 3. İlerleme hızı 16 mm/dak, iş mili devri 200 devir/dak, kesme derinliği 0.35 mm ve radyal dalma oranı $\% 50$ kesme parametrelerınde işlenmiş numune 


\subsection{Varyans Analizi}

Optimum kesme parametreleri tanımlandıktan sonra, her kesme parametresinin katkı yüzdesi göreceli varyanslarını karşılaştırarak hesaplandı. Her parametrenin karelerinin toplamının ve toplam karelerin toplamının hesaplanması aşağıdaki gibi yapılmış$\operatorname{tir}[14]$ :

$$
\begin{aligned}
S S_{P_{i}} & =\sum_{i=1}^{K_{A}}\left(\frac{A_{i}^{2}}{n_{A_{i}}}\right)-\frac{\left(\sum_{i=1}^{N} x_{i}\right)^{2}}{N} \\
S S_{T} & =\sum_{i=1}^{K_{A}} x_{i}^{2}-\frac{\left(\sum_{i=1}^{N} x_{i}\right)^{2}}{N} \\
S S_{E} & =\left(S S_{T}-\sum_{i=1}^{K_{A}} S S_{P_{i}}\right)
\end{aligned}
$$

burada $A_{i}$ her değer için ortalama pürüzlülüğü, $n_{A i}$ değer sayısını, $x_{i}$ her deneydeki pürüzlülük değerini, N deney sayısını ve k_A parametre sayısını temsil eder. Toplam serbestlik derecesinin $(\mathrm{Ft})$ ve her değerin serbestlik derecesinin (Fi) hesaplanması için sırasıyla aşağıdaki denklemler kullanılmıștır:

$F_{i}=x_{i}-1$,

$F_{t}=N-1$,

Serbestlik derecesi hatasını belirlemek için denklem-7 aşağıdaki gibi uygulanmıştır:

$$
F_{e}=F_{t}-\sum_{i=1}^{K_{A}} F_{i}
$$

Her bir parametrenin varyansı $\left(V_{P i}\right)$ ve hata varyansı $\left(V_{e}\right)$ denklem-8 ve denklem9'daki gibi değerlendirilmiştir:

$$
\begin{aligned}
& V_{P_{i}}=\frac{S S_{P_{i}}}{F_{i}} \\
& V_{e}=\frac{S S_{e}}{F_{e}}
\end{aligned}
$$

Son olarak, her parametrenin $\left(P_{P i}\right)$ katkı yüzdesi aşağıdaki gibi hesaplanmıştır:

$$
{ }^{{ }^{\prime} P_{i}}=\frac{S S_{P_{i}}}{S S_{T}},
$$


Tablo 5. Varyans Analiz Sonucu Hesaplanan Parametrelerin Katı Yüzdesi

\begin{tabular}{|l|c|c|c|}
\hline & $\mathbf{F}$ & $\mathbf{S}$ & $\mathbf{P} \%$ \\
\hline$P_{1}:$ İlerleme hızı & 2 & 0.0070 & 6.98 \\
\hline$P_{2}:$ İş mili devri & 2 & 0.0238 & 23.67 \\
\hline$P_{3}:$ Kesme derinliği & 2 & 0.0204 & 20.23 \\
\hline$P_{4}:$ Radyal dalma oranı & 2 & 0.0494 & 49.12 \\
\hline Hata & 0 & 0.0000 & 0.000 \\
\hline Toplam & 8 & 0.1006 & 100.0 \\
\hline
\end{tabular}

Denklem-2 - Denklem-10 kullanılarak her bir parametrenin ağırlık katkı yüzdesi Tablo 5 'te gösterildiği gibi hesaplanmıştır.

\section{SONUÇ VE TARTIŞMA}

Bu çalışmada ilerleme hızı, iş mili devri, kesme derinliği ve radyal dalma oranı gibi parametrelerin işlenen yüzeyin pürüzlülüğü üzerindeki etkisi incelenmiştir. Sinyalgürültü $(\mathrm{S} / \mathrm{G})$ oranı, varyans analizi ve deney tasarımı gibi Taguchi yöntemleri sayesinde, frezeleme süreci sonrasında oluşan işlenmiş yüzey kalitesini iyileştirmek için seçilen kesme parametrelerin optimal seviyeleri araştırılmıştır. Sonuç olarak bu deneyler için radyal dalma oranın diğer parametrelere nazaran daha etkili olduğu ve ardından kesme derinliği, iş mili devri, ilerme hızının geldiği gözlemlenmiştir. En iyi yüzey kalitesi belirlenen optimum parametre seçiminde elde edilmiş olup, en yüksek $\mathrm{S} / \mathrm{G}$ seviyesi veren ilerleme hız1 $16 \mathrm{~mm} /$ dak, iş mili devri $200 \mathrm{devir} /$ dak, kesme derinliği $0.35 \mathrm{~mm}$ ve radyal dalma oranı \%50 değerlerinde elde edilmiştir. Ayrıca her kesme paramentresinin katkı yüzdesi de belirlenmiştir. Sonuç olarak yüzey pürüzlülüğü üzerinde en yüksek katkı oranının radyal dalma oranı parametresine ait olduğu görülmüştür. Taguchi optimizasyon yöntemini kobalt bazlı alaşımın optimum yüzey pürüzlülüğü bulmak için kullanan Bağcı ve Aykut [7] da benze bir şekilde kesme hızı, ilerleme ve kesme derinliği parametrelerinin yüzey pürüzlülüğü üzerinde kayda değer etkisinin oldüğünü belirtmişlerdir. Aynı zamanda, Kuram ve Özçelik [8] istatistiksel yöntemlerin optimum kesme parametrelerini, takım aşınmasını ve kesme kuvvetlerini öngörmek için hızlı, effektif ve endüstriyel uygulamalar için daha kullanışlı bir yol olduğunu belirtmişlerdir.

\section{TEŞEKKÜRLER}

Yazar Azma Mould Manufacturing Industry şirketine deney ve ölçümlerde yaptıkları desteklerinden ötürü teşekkürlerini sunar. 


\section{KAYNAKÇA}

1. Montgomery, D., Altintas, Y. 1991. "Mechanism of Cutting Force and Surface Generation in Dynamic Milling”, Journal of Engineering for Industry, 113(2), pp. 160-168. DOI: 10.1115/1.2899673

2. Ehmann, KF., Hong, MS. 1994. "A Generalized Model of the Surface Generation Process in Metal Cutting”, CIRP Annals - Manufacturing Technology, 43, pp. 483486. DOI: 10.1016/S0007-8506(07)62258-6

3. Layegh, K., SE, Lazoglu, I. 2017. "3D surface topography analysis in 5-axis ballend milling”, CIRP Annals - Manufacturing Technology, 66, pp. 133-136. DOI: 10.1016/j.cirp.2017.04.021

4. Altintas, Y., Budak, E. 1995. "Analytical Prediction of Stability Lobes in Milling", CIRP Annals - Manufacturing Technology, 44, pp. 357-362. DOI: 10.1016/S00078506(07)62342-7

5. Munoa, J., Beudaert, X., Dombovari, Z., Altintas, Y., Budak, E., Brecher, C., Stepan, G. 2016. "Chatter suppression techniques in metal cutting", CIRP Annals Manufacturing Technology, 65, pp. 785-808. DOI: 10.1016/j.cirp.2016.06.004

6. Zahoor, S., Mufti, NA., Saleem, MQ., Mughal, MP., Qureshi, MAM, 2017. "Effect of machine tool's spindle forced vibrations on surface roughness, dimensional accuracy, and tool wear in vertical milling of AISI P20", International Journal of Advanced Manufacturing Technology, 89, pp. 3671-3679. DOI: 10.1007/s00170016-9346-1

7. Bagci, E., Aykut, S. 2005. "A study of Taguchi optimization method for identifying optimum surface roughness in CNC face milling of cobalt-based alloy (stellite 6)", International Journal of Advanced Manufacturing Technology, 29, pp. 940-947. DOI: $10.1007 / \mathrm{s} 00170-005-2616-y$

8. Kuram, E., Ozcelik, B. 2016. "Micro-milling performance of AISI 304 stainless steel using Taguchi method and fuzzy logic modelling”, Journal of Intellegent Manufacturing, 27, pp. 817-830. DOI: 10.1007/s10845-014-0916-5

9. Costa, D., Belinato, G., Brito, T., Paiva, A., Ferreira, J., Balestrassi, P. 2017. "Weighted principal component analysis combined with Taguchi's signal-to-noise ratio to the multiobjective optimization of dry end milling process: a comparative study", The Journal of the Brazilian Society of Mechanical Sciences and Engineering, 39, pp. 1663-1681. DOI: 10.1007/s40430-016-0614-7

10. Manav, C., Bank, HS., Lazoglu, I. 2013. "Intelligent toolpath selection via multicriteria optimization in complex sculptured surface milling", Journal of Intellegent Manufacturing, 24(2), pp. 349-355. DOI: 10.1007/s10845-011-0596-3

11. Arruda, EM., Paiva, AP., Brandão, LC., Ferreira, JR. 2019. "Robust optimisation of surface roughness of AISI H13 hardened steel in the finishing milling using 
ball nose end mills", Precision Engineering, 60, pp. 194-214. DOI: 10.1016/j.precisioneng.2019.07.013

12. Mia, M., Al Bashir, M., Awal, Khan MD., Dha,r NR. 2017. “Optimization of MQL flow rate for minimum cutting force and surface roughness in end milling of hardened steel (HRC 40)", International Journal of Advanced Manufacturing Technology, 89, pp. 675-690. DOI: 10.1007/s00170-016-9080-8

13. Amer, Y., Moayyedian, M., Hajiabolhasani, Z. and Moayyedian, L. 2013. "Improving injection moulding processes using experimental design", (Doctoral dissertation, WASET-World Academy of Science Engineering a). DOI: 10.5281/zenodo.1062096

14. Moayyedian, M., Mamedov, A., Derakhshandeh, JF., Elkattan, A. 2020, "Determination of Significant Parameters and Their Contributions In Temperature Measurement using Analysis of Variance and Taguchi Method", International Journal of Engineering Research and Technology, 9(10), pp.790-796. DOI: 10.17577/IJERTV9IS100264 